\title{
Summary of the Demonstration and Poster Track
}

\author{
Miriam Fernández ${ }^{1}$ and Vanessa López ${ }^{2}$ \\ 1 Knowledge Media Institute \\ The Open University, UK \\ 2 IBM Dublin
}

The demonstration and poster track is an opportunity for researchers and practitioners to present their innovative prototypes, practical developments, on-going projects, lessons learned and late-breaking results. This year we had a very exciting track with thirty-five poster and thirty-two demo submissions. All poster and demonstration papers were peer reviewed by at least 2 reviewers, resulting in twenty accepted posters and twenty-four accepted demos.

Posters and demos give a glimpse to where the Semantic Web is heading. This year they spanned a broad set of topics over which we can highlight the topic of semantic search: linked data indexing, exploratory search over linked data, spoken search over linked data, cross-lingual querying over linked data, federated SPARQL queries and visual query editors. Other less frequent topics presented this year are relation discovery over linked data, facts discovery over linked data, ontologies for the academic domain, ontologies for the music domain, solutions for big data and semantic analyses of the social web.

All posters and demos were presented and voted on by the ESWC audience. The best ESWC 2013 poster was awarded to Tobias Käfer and colleagues for their work "Exploring the dynamics of Linked Data". In this work the authors proposed the Linked Data Observatory, 8 months of weekly collected linked data documents used to study the dynamics and evolution of linked data. The best ESWC 2013 demo award was given to two different works: "Sextant: Browsing and Mapping the Ocean of Linked Geospatial Data" by Charalampos Nikolaou et al. as well as "Exploratory search on the top of DBpedia chapters with the discovery hub application" by Nicolas Marie et al. The first demo is a webbased tool that enables exploration of linked geospatial data and supports the creation, sharing, and collaborative editing of thematic maps. The second demo is an exploratory search engine that helps users to explore topics of interest.

All posters and demonstrations are included in this volume. Demonstration papers are up to 5 pages long, poster papers are shorter with 2 pages maximum.

We hope you enjoy each and one of them! 